L.Y. Beeker, Adam M. Pringle, Joshua M. Pearce. Open-source parametric 3-D printed slot die system for thin film semiconductor processing. Additive

Manufacturing 20 (2018) 90-100. https://doi.org/10.1016/j.addma.2017.12.004 , open hardware source code: https://osf.io/m2zqk/

\title{
Open-source Parametric 3-D Printed Slot Die System for Thin Film Semiconductor Processing
}

\author{
L. Y. Beeker ${ }^{1}$, Adam M. Pringle ${ }^{1}$, Joshua M. Pearce ${ }^{1,2}$
}

1. Department of Materials Science \& Engineering, Michigan Technological University, Houghton MI

2. Department of Electrical \& Computer Engineering, Michigan Technological University, Houghton MI

\begin{abstract}
Slot die coating is growing in popularity because it is a low operational cost and easily scaled processing technique for depositing thin and uniform films rapidly, while minimizing material waste. The complex inner geometry of conventional slot dies require expensive machining that limits accessibility and experimentation. In order to overcome these issues this study follows an open hardware approach, which uses an open source 3-D printer to both fabricate the slot die and then to functionalize a 3-D slot die printing system. Polymer materials are tested and selected for compatibility with common solvents and used to fabricate a custom slot die head. This slot die is then integrated into a 3-D printer augmented with a syringe pump to form an additive manufacturing platform for thin film semiconductor devices. The full design of the slot die system is disclosed here using an open source license including software and operational protocols. This study demonstrates that functional lab-grade slot dies may be 3-D printed using low-cost open source hardware methods. A case study using $\mathrm{NiO}_{2}$ found an RMS value $0.486 \mathrm{~nm}$, thickness of 17 to 49nm, and a maximum optical transmission of $99.1 \%$, which shows this additive manufacturing approach to slot die depositions as well of fabrication is capable of producing viable layers of advanced electronic materials. Using this method, a cost savings of over $17,000 \%$ was obtained when compared to commercial slot die systems for laboratories.
\end{abstract}

\section{Keywords: slot die, 3-D printing, thin film, semiconductor, RepRap, open hardware}

\section{Introduction}

Slot die coating developed in the 1950s by Beguin improved the fabrication process of photographic films and papers [1]. It provides a scalable and cost-efficient means of depositing thin, uniform films rapidly, while minimizing material waste [2]. During the slot die coating process, liquid is pumped from a reservoir through a thin slot, where it is then deposited onto a substrate. An advantage of this method is that the film thickness is predetermined by well controlled parameters. The wet thickness of the deposited layer can be determined by the ratio of the flow rate of the liquid and the coating speed [3]. Many alternative methods such as vacuum deposition and spin coating provide relatively low yields at a higher cost [4,5]. Slot die coating is becoming a favored process due to its low operational cost and potential to be used in large-scale mass manufacturing such as roll to roll processing [2]. Current applications of slot die processing include depositing precise films and electrodes in many devices including solar cells [6], lithium ion batteries [7], and fuel cells [8]. 
L.Y. Beeker, Adam M. Pringle, Joshua M. Pearce. Open-source parametric 3-D printed slot die system for thin film semiconductor processing. Additive Manufacturing 20 (2018) 90-100. https://doi.org/10.1016/j.addma.2017.12.004 , open hardware source code: https://osf.io/m2zqk/

Stainless steel, commonly used to fabricate slot dies [9], is a difficult material to machine partially due to its high strength and work hardening rate [10]. This requires machine shops to be specially equipped for the fabrication of these parts. Furthermore, the complex inner geometry of a slot die requires thetwo halves of the die be machined separately. These restrictions contribute to the high prices of currently available slot dies. For example, a standard 50-millimeter slot die costs \$3,950, which economically restricts access to rapid development of the technology at the lab level [11]. 3-D printing a slot die head has been attempted in the past, however, no models have been released for consumers [12].

To overcome these issues this study follows an open hardware approach [13], which uses a selfreplicating rapid prototyper (RepRap) Delta model 3-D printer [14-16] as a 3-D stage [17,18] to fabricate customized parametric slot die systems. First, a method of testing various 3-D printable polymers for viability with slot dies processing using common solvents (water, ethanol and isopropanol) is described and used to determine appropriate materials. A slot die is then fabricated from the best tested material for matched solvents and a set of controlled prints are run to find the minimum reliable die gap with this printing system. This slot die is then integrated into a 3-D printer augmented with a syringe pump to form an additive manufacturing platform for thin film semiconductor devices. The full design of the slot die system is disclosed here using an open source license including software and operational protocols. A case study example is presented and the material properties of the resultant film are tested and compared to other manufacturing techniques. Finally, this approach is discussed and conclusions are drawn about the viability of distributed manufacturing with slot die systems.

\section{Methods}

\subsection{Slot Die Design}

The slot die was modeled using the open source software, OpenSCAD, a script-based, parametric CAD (computer aided design) program possessing powerful 3-D modeling capabilities [19]. The parametric nature of the code allows the geometries of the slot die to be easily altered. The 3-D slot die design contains basic features that conventional slot dies encompass. The feed port, distribution chamber, and slot gap were modeled from slot dies described in the literature [3,9,20]. A slot gap of $200 \mu \mathrm{m}$ was chosen for this application because similar dimensions have been successfully used in the literature $[3,20]$. Distribution chambers are oriented along the width of the web and typically extend to the end of the slot gap [9]. A single center fed cavity with a uniform teardrop cross section was selected for this application to simplify geometries and improve print quality. An isometric wire frame view of the slot die design is shown in Fig. 1.

The OpenSCAD script allows for various design features to be incorporated into the design of the slot die following advanced geometries previously investigated [20-26]. Dimensional changes to the body of the die, along with the addition of internal features, create the potential for the fabrication of dual cavity slot dies. Dual cavity slot dies will commonly utilize a tapered, coat hanger inner cavity [27]. These geometries are entirely printable; however, print settings must be selected to ensure the inner slot is unimpeded by filament stringing by ensuring that filament retractions are adequate, or temperature is correctly set. Slot dies with flexible lips to adjust coating thickness $[9,28]$ need an appropriate polymer and there have been new flexible filaments commercialized, which need to be studied in future work. 
L.Y. Beeker, Adam M. Pringle, Joshua M. Pearce. Open-source parametric 3-D printed slot die system for thin film semiconductor processing. Additive

Manufacturing 20 (2018) 90-100. https://doi.org/10.1016/j.addma.2017.12.004 , open hardware source code: https://osf.io/m2zqk/

An alternative option provided by this method is to alter the OpenSCAD code and fabricate a separate slot die with different dimensions. Additionally, the tip of a slot die may be easily printed with varied configurations following Harris et al. [21]. The slopes of upstream die geometries affect coating defects such as ribbing and air entrapment [29]. Minimal adjustments to the design at the code level enable the user to control the slopes to best suit the application.

Fig. 1. OpenSCAD wireframe rendering of parametric slot die design.

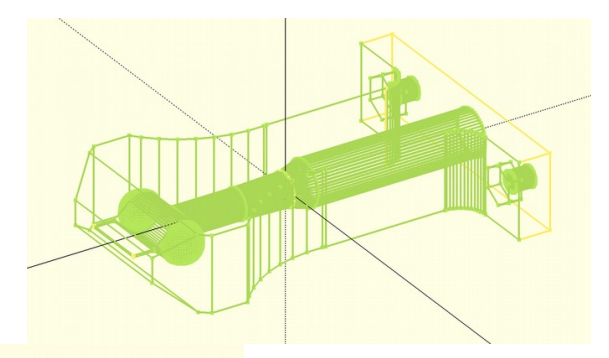

Fig. 2. Slot die schematic with labeled geometries

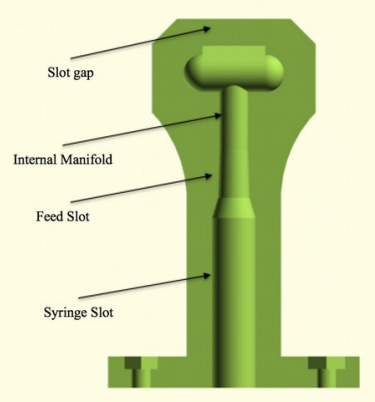

\subsection{Slot Die Materials Selection}

Slot dies are subjected to a wide range of chemical solutions. As a result, slot dies are commonly manufactured in stainless steel due to its low reactivity [9]. Although it is possible to 3-D print steel, commercial systems still have high costs [30], and low-cost RepRap 3-D printing of metal [31-35] can not yet reach sufficient resolution for the precise micron tolerances needed for this application. However, polymer fused filament fabrication (FFF) may accomplish the necessary geometric complexities. To determine which polymer would be used to create the slot die, the absorptive properties of multiple filament types were determined by testing commonly available filaments including: polylactic acid (PLA), polypropylene (PP), nylon, polyethylene terephthalate glycolmodified (PETG), acrylonitrile butadiene styrene (ABS), ninjaflex (NF), polycarbonate (PC), and highimpact polystyrene (HIPS). Tested dimensions were $1 \mathrm{~cm}$ in length and $1.75 \mathrm{~mm}$ in diameter. The change in mass and dimension were recorded using a digital scale and (+/- 0.0001g) digital calipers (+/$0.01 \mathrm{~mm}$ ) and analyzed to determine the appropriate polymer.

The filament sections were placed in the following solvents: water, ethanol, titanium ink (ethanolisopropanol based), and nickel ink (ethanol based) for a duration of 144 hours (six days). This time frame was chosen because it far exceeded, by four magnitudes, the average print time of three minutes for semiconductor thin film prints. The titanium and nickel inks were selected for viable application to test printed slot die capability. The titanium ink was created via a 1-125-19-125 part mixture of $\mathrm{HCl}(0.33 \mathrm{~mL})$-isopropanol(41.50mL)-titanium(IV) isopropoxide(6.22mL)-ethanol(41.50mL) with a 
L.Y. Beeker, Adam M. Pringle, Joshua M. Pearce. Open-source parametric 3-D printed slot die system for thin film semiconductor processing. Additive Manufacturing 20 (2018) 90-100. https://doi.org/10.1016/j.addma.2017.12.004 , open hardware source code: https://osf.io/m2zqk/

verification of 3-4pH to create a clear solution [36]. The nickel ink was created via a 1:1 mol ratio of nickel acetate tetrahydrate (NAT,2.01g) : monoethanolamine (MEA, 0.49mL) with the NAT dissolved in ethanol (79.51mL) [37]. All solutions were purchased from Sigma Aldrich with the exception of the nickel acetate tetrahydrate powder which was purchased from Acros Organics. The solution was then heated at $70^{\circ} \mathrm{C}$ for 4 hours with stirring and after 30 minutes the solution turned a deep green [37].

For this particular case study, the pressures the slot die must obtain are modest because of the low viscosity of the solution. However, to determine the viability for this approach to high viscosity fluids the maximum allowable pressure within the slot die was approximated via Barlow's formula [37,38]. The minimum dimensions were assumed and the tensile strength of PETG (49MPa) [39,40] was halved to account for printing influenced weaknesses, which yielded a value of 18MPa. Fluids of typical viscosities would not pose a threat to the integrity of the die if a slot thickness $200 \mu$ m were utilized [41]. Finite element analysis may be utilized in the future to obtain a more precise value for a given slot die geometry.

\subsection{Slot Die Fabrication}

The slot die was printed by an Athena version of the open source MOST delta RepRap 3-D printer [42] initially in poly lactic acid (PLA) from Hatchbox for geometric prototyping, which was later changed to poly(ethylene glycol-co-1,3 cyclohexanedimethanol terephthalate) (PETG , from eSUN 3D) due to solution resistance testing. The STL (stereolithography) file was exported from OpenSCAD and imported into Cura [43], an open source slicing program, which allows printing parameters to be determined. This presents a completely open source hardware and software tool chain. The delta RepRap uses standard FFF, where a continuous thermoplastic filament material is fed from a large spool, through a moving, heated extruder head.. A vertical printing orientation, where the slot gap is printed last, was determined to be most appropriate for the design. Due to the geometry, any other orientation would create overhangs, which are detrimental to the printing precision. This orientation also allowed for a sufficient area to be utilized as a base so adhesion to the print bed was adequate when combined with a brim option in Cura. After prototyping the slot die the final print settings were determined, the most important settings are summarized in Table 1. The internal geometry for the infill density was set to be the standard, lines crossing at 90 degrees internally. The slot die should not be under any significant stresses, so the infill density may be reduced to preserve material. From an additive manufacturing perspective, similar shell thickness and bottom/top thicknesses are critical for a uniformly strong part. A print speed of $30 \mathrm{~mm} / \mathrm{s}$ was chosen in combination to a layer height of 100 microns in order to maximize precision, primarily for the slot die top gap, and a slower bottom speed was necessary to provide proper adhesion to the print bed. The slot dies were printed one at a time to eliminate stringing between parts and improve quality. Because of this, the time the nozzle remained close to a section of the part during printing was extended, thus the temperature was reduced to $195^{\circ} \mathrm{C}$ from $215^{\circ} \mathrm{C}$ normally used for PETG to prevent quality loss due to excess heat.

The complete settings are in the supporting information the G-code were exported from Cura and imported into the firmware, Franklin [44,45]. The slot die can be printed in approximately three hours at optimal settings, with a prototype low quality taking around 55 minutes to complete. The accuracy of the printed gaps was determined by printing a series of seven unique slot die tops with progressively 
L.Y. Beeker, Adam M. Pringle, Joshua M. Pearce. Open-source parametric 3-D printed slot die system for thin film semiconductor processing. Additive Manufacturing 20 (2018) 90-100. https://doi.org/10.1016/j.addma.2017.12.004 , open hardware source code: https://osf.io/m2zqk/

smaller gaps from 400 microns to 100 microns until the gap became inconsistent. The gaps were imaged with a digital microscope and then six measurements were averaged for the tips being quantified with imageJ [46].

Table 1. Essential Cura settings for printing a slot die in PETG used in this study.

\begin{tabular}{|c|c|c|}
\hline Setting & Units & Value \\
\hline Layer Height & $\mu \mathrm{m}$ & 100 \\
\hline Shell Thickness & $\mathrm{mm}$ & 1.0 \\
\hline Bottom/Top Thickness & $\mathrm{mm}$ & 1.0 \\
\hline Nozzle Size & $\mathrm{mm}$ & 0.5 \\
\hline Print Speed & $\mathrm{mm} / \mathrm{s}$ & 30 \\
\hline Top/Bottom Speed & $\mathrm{mm} / \mathrm{s}$ & 15 \\
\hline Printing Temperature & ${ }^{\circ} \mathrm{C}$ & 195 \\
\hline Minimal Layer Time & $\mathrm{sec}$ & 14 \\
\hline Infill density & $\%$ & 35 \\
\hline
\end{tabular}

\subsection{Slot Die Implementation}

The slot die was made to be compatible with a Prusa Mendel i1 RepRap 3-D printer [47], modified for syringe pump [48] use following earlier work in wax 3-D printing [49]. The mounting system was derived from the x-carriage of the Prusa Mendel I1 [47] open-source 3-D printable library. Four holes were added to the $\mathrm{x}$-carriage to mount the syringe pump, two more were added to mount the slot die, all with $3 \mathrm{~mm}$ screws and nuts. With the slot die mounted a $1 \mathrm{~mL}$ syringe is then loaded into the slot die without the needle tip attached. The syringe pump (through Franklin) pulls the syringe end extending it and allows air to enter the syringe itself. Then through Franklin (the function to extrude filament) the syringe is pressed and solution enters the internal geometries of the slot die. This process continues until a meniscus is formed at the end of the slot die. This indicates that the slot die is primed for printing. The fully modified 3-D printer is shown in Fig. 3. Utilizing Franklin, final adjustments are made to calibrate the primed slot die to the surface of the substrate to be printed upon (200 microns for this study), in this case a laboratory microscope slide (soda lime glass). Running a Gcode line, allows the slot die to move as the print head of the 3-D printer. Modifying the extrusion rate in Franklin or the flow rate in Cura allows the volumetric flow rate to be adjusted for specific film thicknesses.

Additionally, the motor speed can be adjusted in Franklin or Cura to change the velocity of the slot die during printing. A case study example is presented for nickel oxide, which is used in semiconducting devices, solar cell architectures, thermo-electric devices, and laser materials [50]. For this experiment, a print speed of $30 \mathrm{~mm} / \mathrm{s}$ and an extrusion rate of 1000\% was utilized to produce nickel acetate tetrahydrate thin films to be decomposed into nickel oxide (NiO) via a thermal heat treatment at $275^{\circ} \mathrm{C}$ for 45 minutes in atmosphere. FFF 3-D printers use linear extrusion to control the volume of filament extruded via normal printer operation. For the syringe pump adaptation, in order to have reliable 
precision in depositions a $1 \mathrm{~mm}$ to $0.01 \mathrm{~mL}$ ratio of linear extrusion to liquid extrusion for a $1 \mathrm{~mL}$ syringe was set. This relationship was determined via experimentation by adjusting the coupling setting on the extrusion motor to be 700 steps $/ \mathrm{mm}$ for a $5 \mathrm{~mm}$ diameter threaded rod. Utilizing custom gcode, a $0.2 \mathrm{~mm}$ linear extrusion $(0.002 \mathrm{~mL}$ liquid extrusion) was used for each layer in order to provide consistent layer thickness. Thus, $0.002 \mathrm{~mL}$ per pass is the base extrusion. It is also important to note that a pre-print extrusion of $5 \mathrm{~mm}$ or $0.01 \mathrm{~mL}$ was used in order to wet the slot die and facilitate liquid transfer from the syringe to the substrate.

Fig. 3. X-carriage modified for slot die capability.

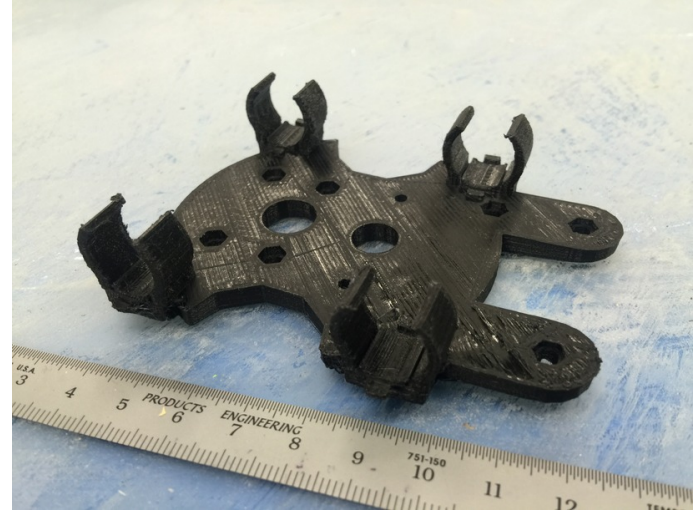

Fig. 4. Prusa Mendel i1 RepRap 3-D printer fully modified for slot die deposition.

\subsection{Thin Film Characterization}

Several testing methods were employed to measure the effectiveness and characterize the thin films created including atomic force microscopy, spectroscopic ellipsometry and transmission measurements.

The NiO thin films were measured and recorded on a VEECO Nanoscope II AFM. Surface roughness measurements were done with a scan size of $1 \times 1$ $\mu \mathrm{m}$ and scanning rate of $0.505 \mathrm{~Hz}$. Profile measurements were conducted with a scan size of $10 \times 10 \mu \mathrm{m}$ and a scanning rate of $1.001 \mathrm{~Hz}$. Thickness of the thin film was measured via a scratch method with a scan size dimension of $110 \mathrm{x} 10 \mu \mathrm{m}$ and a scanning rate of $0.250 \mathrm{~Hz}$. Additional settings include an integral gain of 0.100 , a proportional 
L.Y. Beeker, Adam M. Pringle, Joshua M. Pearce. Open-source parametric 3-D printed slot die system for thin film semiconductor processing. Additive

Manufacturing 20 (2018) 90-100. https://doi.org/10.1016/j.addma.2017.12.004 , open hardware source code: https://osf.io/m2zqk/

gain of 0.150 , and an amplitude set point of $1.300 \mathrm{~V}$. The scratch methodology included making a light pass with an X-ACTO knife over both the glass substrate and deposited $\mathrm{NiO}$ thin film. A relatively slower scanning rate was critical for the thickness measurement because of the sharpness of the created scratch in order to ensure detection. Sites were chosen for thickness measurement when the scratch only impacted the thin film and not the glass substrate to account for scratch to scratch stress variability.

To verify the thickness measurements, a J.A. Woollam variable-angle spectroscopic ellipsometer (VASE) was used. It is equipped with a HS-190 monochromator and HTC - 100 heat stage. NiO layer measurements were conducted in the wavelength range of $300-1000 \mathrm{~nm}$. A weak vacuum was utilized to hold the sample in place. Standard operation procedures were then used for data collection.

Transmittance was conducted utilizing an Ocean Optics USB2000+ spectrophotometer as a detection device via the SpectraSuite software. The light source used was an Ocean Optics DH-2000-CAL. It is a UV-VIS-NIR light source, and the halogen lamp was active for testing. The detection range of 400$1000 \mathrm{~nm}$ was measured for all samples. As the samples were coated on glass slides a 3-D printed part [51] was modeled and printed to secure both the sample slide as well as the fiber optic cables transmitting the light. Measurements were conducted under a black box with light and dark reference being taken with a clean glass slide substrate.

\section{Results and Discussion}

\subsection{Slot Die Material Selection}

All samples were submerged in solutions of water, and ethanol, titanium isopropoxide (primarily isopropanol), and nickel acetate tetrahydrate dissolved in ethanol. Minimal effects were observed on the PETG and PP filaments, while PLA showed degradation, as can be seen in Table 2. The chemistry of PLA, PP, and PETG is compared in Fig. 5.

Fig. 5. Chemistry differences between PP, PLA, and PETG.

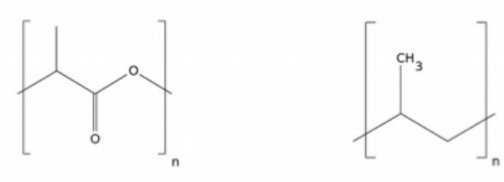

Polylactic acid(PLA)

Polypropylene(PP)

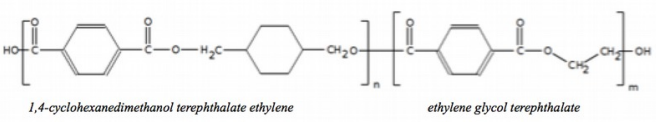

In general, a material is soluble in a solvent, like water, if there is a soluble functional group, such as a polar oxygen molecule, per five non-soluble backbone atoms. In this case, the backbone atoms are a non-polar carbon chain. The polymers in Fig. 5 all have a carbon backbone which promotes high resistivity to water. PLA demonstrates a two to three, soluble to non-soluble atom ratio making it the least resistive to water within this group. Furthermore, lactide polymers are well known to undergo hydrolysis, so mass loss due to interaction with water was to be expected. This polymer was added for comparison due to its wide usage for prototyping in additive manufacturing, particularly among FFF 3-D printer users. This is demonstrated by data gathered here shown in Table 2 . The methyl group within the monomer of PP and PLA, as well 
L.Y. Beeker, Adam M. Pringle, Joshua M. Pearce. Open-source parametric 3-D printed slot die system for thin film semiconductor processing. Additive

Manufacturing 20 (2018) 90-100. https://doi.org/10.1016/j.addma.2017.12.004 , open hardware source code: https://osf.io/m2zqk/

as, the cyclohexane and benzene rings found in PETG further promote the hydrophobic nature of these polymers by both being non-polar and introducing steric hindrance, which creates a general decrease in reactivity due to the bulky nature of these molecules reducing non-similar bonding type interactions, even though the PETG co-polymer has hydroxyl groups, esters, and ketones, all which demonstrate hydrogen bonding. This bonding would normally allow solubility in both water and ethanol, but since the functional groups are outweighed molecularly by the non-polar bonded carbon-hydrogen backbone, PETG is hydrophobic. Given the negligible amount of degradation of these three polymers over the testing period, these polymers can be considered stable when used around both water and ethanol for extended durations.

It is well understood that to be soluble in a solution the solute must have similar bonding, as well as be easily surrounded by the solvent. However, when placed in an alkaline solution, the ester bond is susceptible to attack by -OH and - $\mathrm{COOH}$ chemical groups [52]. As such, for best use, PLA and PETG should be avoided when basic solutions are required.

PP, PLA and PETG are biodegradable [53,54]. Both PETG and PLA have confirmed microorganisms which accelerate degradation [53,55]. Thus, further use of these polymers is not particularly harmful long term for the environment.

Nylon demonstrates the most severe mass change (5.78\%) in water, while Ninjaflex changes (12.58\%) the most when submersed in ethanol. As seen in Table 2, PETG displayed a higher degradation resistance to the solutions investigated. Due to the relatively high coefficient of shrinkage of PP, the mechanical stability of the printed parts becomes difficult to maintain [56]. This is a challenge, although, from these results PP appears promising to investigate in future work, preferably with a heated substrate to reduce shrinkage.

Mass change or dimensional change of a polymer creates problems for applicational use and causes deviations from intentioned properties. The surface of a soluble polymer is first affected by a solution and in this experiment, polymers showing higher mass or radius changes demonstrated a softening of the material surface. It is important to note that this test did not measure the interlayer adhesion which is the backbone of additive manufacturing, but only bulk properties. If printing parameters are not set correctly poor interlayer adhesion may result in a semi-porous printed part. If this is the case, the degradation of a material will be accelerated due to an increase in surface area as the interlayer adhesion breaks down, as well as a significant reduction in the mechanical properties. 
L.Y. Beeker, Adam M. Pringle, Joshua M. Pearce. Open-source parametric 3-D printed slot die system for thin film semiconductor processing. Additive Manufacturing 20 (2018) 90-100. https://doi.org/10.1016/j.addma.2017.12.004 , open hardware source code: https://osf.io/m2zqk/

Table 2. Change in mass of various filaments after 6 days of submersion in water and ethanol trial solutions.

* denotes 3mm diameter filaments

\begin{tabular}{|c|c|c|c|c|}
\hline & \multicolumn{2}{|c|}{ Water } & \multicolumn{2}{c|}{ Ethanol } \\
\hline Filament & $\Delta$ Mass (\%) & $\Delta$ Radius (\%) & $\Delta$ Mass (\%) & $\Delta$ Radius (\%) \\
\hline PLA & -0.33 & 0.95 & 2.14 & -0.38 \\
\hline PP & -0.12 & 0.00 & 0.12 & -0.07 \\
\hline Nylon* & 5.78 & 2.75 & 5.99 & 1.85 \\
\hline PETG & -0.11 & 1.15 & 0.56 & 0.10 \\
\hline ABS* & 0.51 & -0.69 & 3.43 & 0.93 \\
\hline NF & 0.80 & -0.75 & 12.58 & 1.34 \\
\hline PC* & 0.85 & 1.15 & 0.45 & 1.61 \\
\hline HIPS & 0.04 & -0.58 & 0.62 & 0.46 \\
\hline
\end{tabular}

Table 3. Change in mass of final filaments after 6 days of submersion in $\mathrm{NiO}$ and titanium isopropoxide solutions.

\begin{tabular}{|c|c|c|c|c|}
\hline & \multicolumn{2}{|c|}{ NiO Solution } & \multicolumn{2}{c|}{ Titanium Isopropoxide } \\
\hline Filament & $\Delta$ Mass (\%) & $\Delta$ Radius (\%) & $\Delta$ Mass (\%) & $\Delta$ Radius (\%) \\
\hline PP & -0.23 & 0.59 & -0.33 & -0.39 \\
\hline PETG & -0.22 & 0.38 & -0.34 & -0.19 \\
\hline
\end{tabular}

While PP and PETG displayed similar values of mass and radius change within the NiO solution, PETG was determined to be the material to move the study forward. The primary reason for this was the ease of printability of the polymer compared to PP. The titanium isopropoxide solution was tested in parallel to ascertain future work potential.

Next, to determine the minimum gap width the seven as designed slot gap widths were measured.

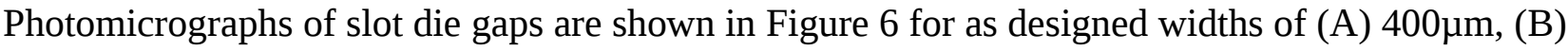
$350 \mu \mathrm{m}$, (C) $300 \mu \mathrm{m}$, (D) $250 \mu \mathrm{m}$, (E) $200 \mu \mathrm{m}$, (F) $150 \mu \mathrm{m}$, and (G) $100 \mu \mathrm{m}$. Averaged experimental widths for tips A-G were $410 \mu \mathrm{m}, 360 \mu \mathrm{m}, 350 \mu \mathrm{m}, 270 \mu \mathrm{m}, 240 \mu \mathrm{m}, 180 \mu \mathrm{m}$, and $140 \mu \mathrm{m}$ respectively. The gap width became inconsistent at 100 microns, which indicates the minimum reliable gap width for this material-device combination. For each size of slot die gap, three printed parts were fabricated and measurements were taken three times on both left, center, and middle sections of the gap. 

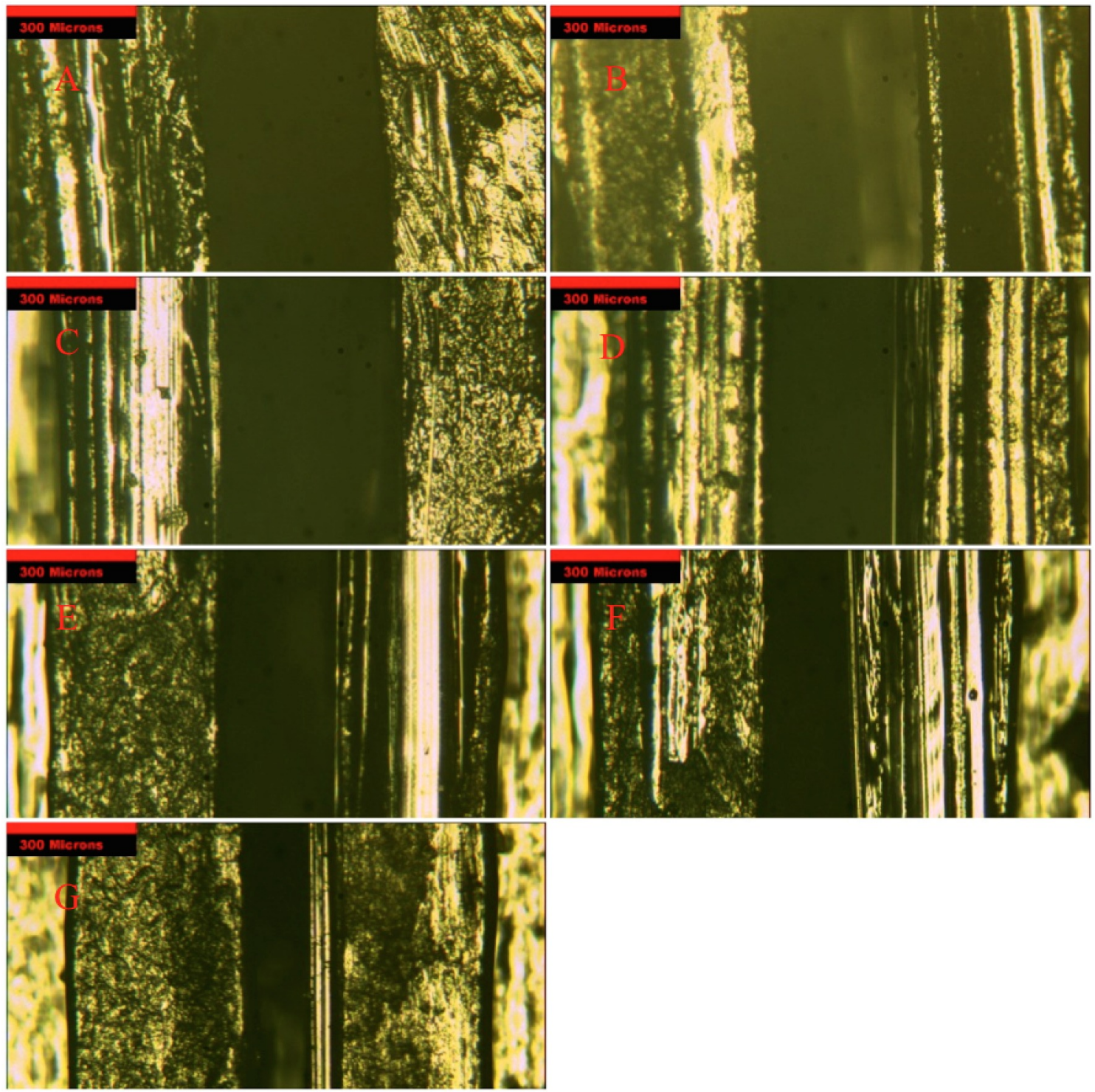

Fig. 6. Photomicrographs of slot die gaps of widths (A) $400 \mu \mathrm{m}$, (B) $350 \mu \mathrm{m},(\mathrm{C}) 300 \mu \mathrm{m}$, (D) $250 \mu \mathrm{m},(\mathrm{E}) 200 \mu \mathrm{m},(\mathrm{F})$ $150 \mu \mathrm{m}$, and (G) $100 \mu \mathrm{m}$. Averaged widths for tips A-G were measured via ImageJ [45] as $410 \mu \mathrm{m}, 360 \mu \mathrm{m}, 350 \mu \mathrm{m}, 270 \mu \mathrm{m}$, $240 \mu \mathrm{m}, 180 \mu \mathrm{m}$, and $140 \mu \mathrm{m}$ respectively.

\subsection{Open source RepRap-based slot die deposition system}

Following an open source protocol, a RepRap-based slot die deposition system was successfully modeled and fabricated as seen in Fig. 7. The source code for the design is available on the open science framework [57]. 
Fig. 7. Top left: 3-D printed slot die (top left) with exposed internals and un-hulled geometries with the corresponding OpenSCAD model (top right). Bottom left: 3-D printed slot die (top left) with exposed internals and hulled geometries with the corresponding OpenSCAD model (bottom right).
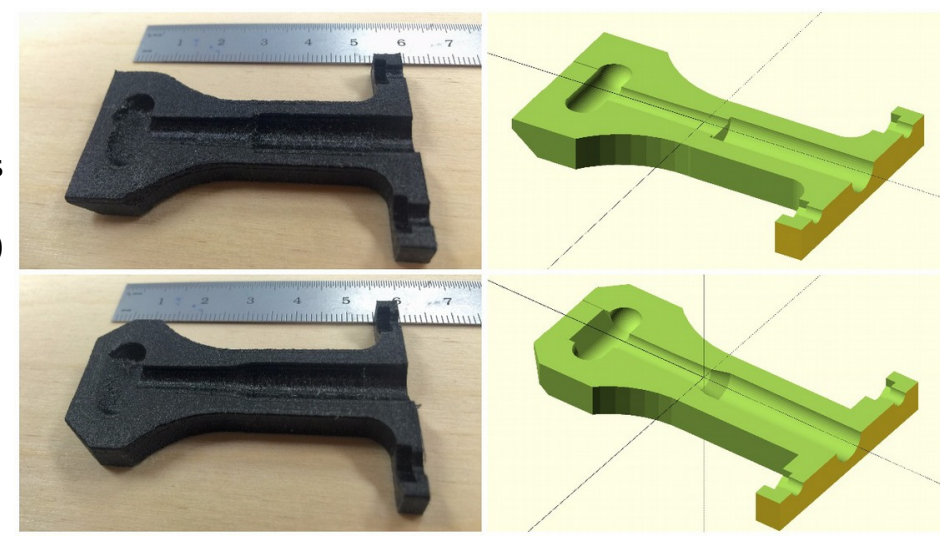

Fig. 8. Final 3-D printed slot die in PETG

Because of the Prusa's optimization for $\mathrm{x}$-directional movement (the two $\mathrm{x}$-carriage guide rods run in the $\mathrm{x}$ direction on the print bed), a 90-degree rotation in the neck of the die was employed to the increase uniformity of thin films. The developed slot die contained a manifold with cross sectional radius of $3 \mathrm{~mm}$. The length was approximately 6 $\mathrm{cm}$, with the width and height at $40 \mathrm{~cm}$ and $12 \mathrm{~cm}$ respectively. The manifold was designed to have a $3 \mathrm{~mm}$ cross-sectional radius and the screw holes are designed for

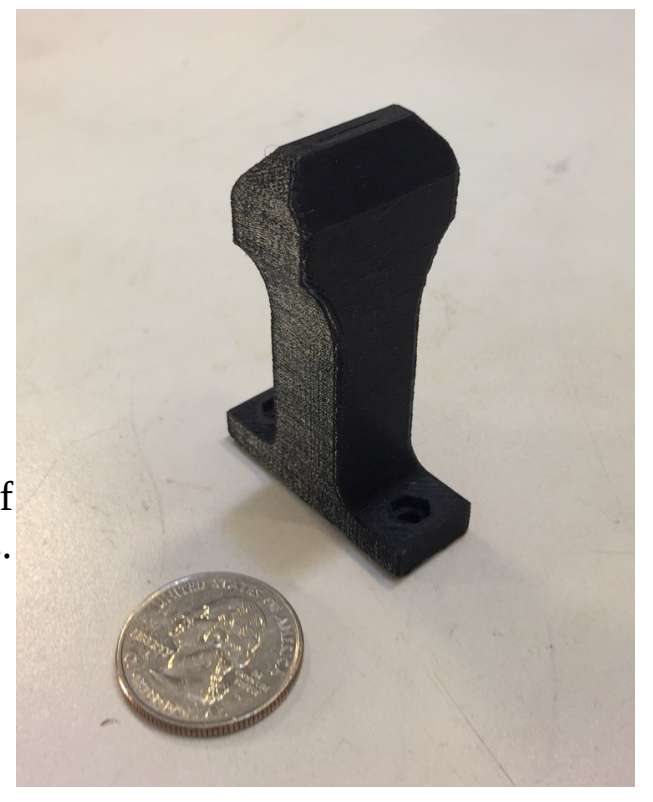
compatibility with m3 screws.

Printing a die with clean (no stringing or bridging) internal geometries was accomplished by use of OpenSCAD's hull feature. As can be seen in Fig. 7, utilizing the hull feature to transition between rough edges allowed smooth transition for the print head preventing stringing or bridging within the internal geometries. It is clear that iterative rapid prototyping provided useful means of implementing design alterations. The final 3-D printed slot die in PETG is shown in Fig. 8.

\subsection{Physical Properties of Deposited Layers}

Fig. 9 demonstrates a representative visual of the surface roughness of the slot die coated NiO layers with varying extrusion rates shown as percentage increases over the base extrusion rate. 
L.Y. Beeker, Adam M. Pringle, Joshua M. Pearce. Open-source parametric 3-D printed slot die system for thin film semiconductor processing. Additive Manufacturing 20 (2018) 90-100. https://doi.org/10.1016/j.addma.2017.12.004 , open hardware source code: https://osf.io/m2zqk/

Fig. 9. 1um x 1um surface plot of $\mathrm{NiO}$ slot coated sample at: A. $100 \%$ Extrusion, $\mathrm{Z}$ of $4 \mathrm{~nm}$ B. $200 \%$ extrusion, $\mathrm{Z}$ of $8 \mathrm{~nm}$ C. $400 \%$ Extrusion, $\mathrm{Z}$ of 6nm, D. $800 \%$ Extrusion, $\mathrm{Z}$ of $15 \mathrm{~nm}$.

The thickness, roughness, and percentage transmission for each of the four samples is summarized in Table 4. In general as the extrusion rate increases (with the slot speed constant) the thickness and roughness increase. Using AFM values, the thinnest sample (A) had a root mean squared (RMS) roughness of $1.66 \mathrm{~nm}$ less than the
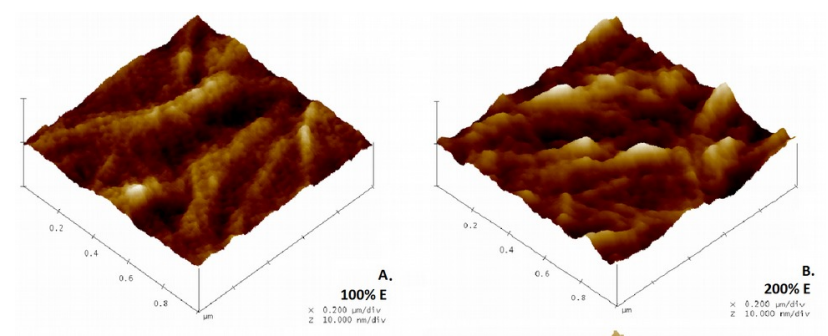
thickest sample (D) for the $1 \times 1 \mu \mathrm{m}$ area. For the larger 10x10 $\mu \mathrm{m}$ area, this difference in roughness increases to $3.059 \mathrm{~nm}$. It is also important to note that sample A is almost three times thinner than sample D (17 nm compared to $49 \mathrm{~nm}$ ). Thus, by simply changing the extrusion rate the surface roughness and thickness may be modified or tuned for a specific application.

The data also shows that as the thickness of the layer increases, the percentage of light transmitted through the sample decreases as would be expected. Sample A displayed a transmission of about 99.1\%, while the sample D showed a slightly lower transmission of about $94.5 \%$. To decrease the error on the transmission measurements three scans were averaged for each measurement and the integration time for each scan was increased to one second. This decreased the deviation in percentage transmission to about 0.6 .

In the literature various methods have been used to generate thin $\mathrm{NiO}$ films including spin-coating [58], pulsed laser deposition (PLD)[59], E-beam [60], and spin-casting [61]. The values for roughness, thickness and percent transmission gained from these methods are displayed in Table 5.

Table 4: Physical characteristics of the printed layers

\begin{tabular}{|c|c|c|c|c|c|c|}
\hline \multirow{2}{*}{\multicolumn{2}{|c|}{$\begin{array}{c}\text { Property } \\
\text { Method (\% Extruded) }\end{array}$}} & \multicolumn{2}{|c|}{$\begin{array}{c}\text { Thickness } \\
\text { (nm) }\end{array}$} & \multicolumn{2}{|c|}{$\begin{array}{l}\text { Roughness } \\
\text { (RMS, nm) }\end{array}$} & \multirow{3}{*}{$\begin{array}{c}\begin{array}{c}\text { Transmission } \\
(\% \mathrm{~T} \pm 0.6)\end{array} \\
\text { Spectrophotometer } \\
99.1\end{array}$} \\
\hline & & Ellipsometer & AFM & $\mathrm{AFI}$ & AFN & \\
\hline \multirow{2}{*}{ Samples } & $\mathrm{A}(100)$ & 32 & 17 & 0.486 & 0.663 & \\
\hline & $\mathrm{B}(200)$ & 29 & 20 & 0.848 & 0.875 & 98.8 \\
\hline
\end{tabular}


L.Y. Beeker, Adam M. Pringle, Joshua M. Pearce. Open-source parametric 3-D printed slot die system for thin film semiconductor processing. Additive Manufacturing 20 (2018) 90-100. https://doi.org/10.1016/j.addma.2017.12.004 , open hardware source code: https://osf.io/m2zqk/

\begin{tabular}{|c|c|c|c|c|c|c|}
\hline \multirow{3}{*}{ Tested } & $\mathrm{C}(400)$ & 29 & 28 & 0.873 & 0.896 & 98.7 \\
\cline { 2 - 6 } & $\mathrm{D}(800)$ & 47 & 49 & 2.146 & 3.722 & 94.5 \\
\hline
\end{tabular}

Table 5: Physical characteristics of NiO thin films by other methods

\begin{tabular}{|c|c|c|c|}
\hline Method & Lowest RMS value (nm) & Thinnest thickness (nm) & Transmission (\%T) \\
\hline Sol - Gel Spin coating & $2.47[58]$ & 28 & 80-92 [62], 95-98\% [63] \\
\hline PLD & $1.0-1.5[59]$ & $5[59],[64]$ & Transparent [64] \\
\hline E-Beam & $1.3[60]$, & $5[60]$ & $92[65]$ \\
\hline Spin-Casting & $0.88[61]$ & $5[61]$ & --- \\
\hline
\end{tabular}

The AFM indicated the thinnest layer deposited to be the lowest extrusion rate at $17 \mathrm{~nm}$ thick, with the thickest layer at $49 \mathrm{~nm}$ thick. Ellipsometry was conducted to verify these measurements. However, the data gathered for the lowest extrusion rate samples, A and B, did not follow the predicted trend for decreasing thickness with less extrusion. The MSE values for the fits are shown in Table 6, a fit of less than 10 is ideal. One potential reason for the thickness measured via ellipsometry for samples A and B not to be less than sample $\mathrm{C}$ is that as the concentration of sample becomes lower, the index contrast between the sample and the substrate also decreases. Thus, the Psi/Delta curves become similar to those for uncoated glass, implying a loss in sensitivity.

Table 6: Fit MSE Values for Ellipsometer measurements

\begin{tabular}{|c|c|}
\hline $\begin{array}{c}\text { Samples Tested (\% } \\
\text { Extruded) }\end{array}$ & Ellipsometer MSE Value \\
\hline $\mathrm{A}(100)$ & 4.613 \\
\hline $\mathrm{B}(200)$ & 2.826 \\
\hline $\mathrm{C}(400)$ & 4.601 \\
\hline $\mathrm{D}(800)$ & 8.443 \\
\hline
\end{tabular}

\subsection{Advantages of 3-D printing slot die systems}

The ability to 3-D print slot dies provides many benefits in the research setting including cost savings, time savings and improved customization and experimental control.

First, costs are significantly reduced when using this method. Commercially available slot dies of comparable sizes typically cost around $\$ 4,000$ [11]. The slot die used in this experiment had a mass of 9 grams and at $\$ 25.95 / \mathrm{kg}$ for filament was produced at a marginal cost less than 25 cents in materials. This is about a 17,000\% decrease in cost. The investment in the 3-D printer, energy used, and labor costs were not included in the comparison. Some points should be clarified for each of these assumptions to show that such a comparison is valid. First, the 3-D printer used in this study was a kit 
L.Y. Beeker, Adam M. Pringle, Joshua M. Pearce. Open-source parametric 3-D printed slot die system for thin film semiconductor processing. Additive Manufacturing 20 (2018) 90-100. https://doi.org/10.1016/j.addma.2017.12.004 , open hardware source code: https://osf.io/m2zqk/

built 3-D printer that costs $\$ 500$ in parts itself and is thus easily amortized printing even a single slot die. Any RepRap class FFF 3-D printer could provide similar results and their prices range from a few hundred to a few thousand dollars, including commercialized fully-assembled and self-bed-leveling and calibrated machines such as the open source Lulzbot Taz 6, which retails for \$2,500 [66]. In addition, most university and industry labs now have access to such 3-D printers (e.g. either in the institution internally or externally at makerspaces [67], hackerspaces [68], fablabs [69,70], and even many libraries [71,72]) and in general the use of the printer is free or set as the cost of the filament with a modest markup. Finally, there are now hundreds of free designs of open source 3-D printable scientific tools, products and components that in general offer savings of $90 \%$ or more over conventional products [73-78]. Thus, the payback for the purchase of a FFF 3-D printer is often rapid for researchers as it can be accomplished with a single print or instrument. After the payback is met the marginal cost of producing a part is the appropriate comparison to the purchase price [79]. For completeness the cost of electricity should also be factored into the distributed manufacturing costs, but has been previously shown to be a small percent of the total cost of a FFF 3-D printed product [80,81], and in general labs do not directly pay their own electricity costs. Lastly, these cost savings assume that there is no labor cost for the 3-D printing. As finding the design made available in this article, customizing it and sending it to a 3-D printer are generally less time intensive than shopping for, ordering and unpacking an equivalent commercial product, the labor costs set to zero is a conservative assumption. Most universities now have at least basic 3-D printing capabilities somewhere on campus. For researchers wishing to fabricate a slot die with no access to 3-D printers locally, an on demand quasi-local 3-D print service can be used, such as MakeXYZ [82]. The costs for a 3-D printing service will be more than the costs of the materials alone. Quotes are available immediately for users in any given area, which enables a high degree of competition so that the overall costs for the slot die will still be several orders of magnitude below the cost of commercial product.

Second, the time spent waiting on the part fabrication is reduced to the length of one print, which was approximately 3 hours. To fabricate these polymer-based slot dies one need only access to a FFF RepRap or similar 3-D printer, and because of the simple installation process, the part can be implemented quickly in both standard systems as well as configuring the same FFF printer into a slot die deposition additive manufacturing system. Industry delivery times for slot dies typically exceed 12 weeks [66]. 3-D printing fabrication methods allow for different dies to be installed quickly for a variety of purposes. Lastly, use of OpenSCAD software allows for the geometries to be quickly changed for different applications. The parametric form of the code allows for simple dimensional modification of the slot gap, slot thickness, and body length. This enables experiments with internal geometries for slot die fabrication, which were prohibitively expensive for traditional materials. Thus, this provides researchers in the thin film industries with a cost-effective method of deposition for solution inks. 3-D printing a slot die also proves to be economical when comparing to vacuum deposition and spin coating [4,5].

In addition, it should be pointed out that the other parts of the experimental setup can also be adjusted as needed. For example, if only a delta-style 3-D printer is available it can be converted to a stage printer [17] and then the syringe pump can be permanently affixed above the printer to obtain the necessary functionality. It should also be noted that the nature of the example here (solar photovoltaic material testing) uses a relatively large amount of low-cost materials. Other research involves depositing expensive materials. In these cases, the nature of the economics of research would demand a smaller syringe to avoid wasting materials. A smaller syringe is easily accommodated by this setup. 
L.Y. Beeker, Adam M. Pringle, Joshua M. Pearce. Open-source parametric 3-D printed slot die system for thin film semiconductor processing. Additive

Manufacturing 20 (2018) 90-100. https://doi.org/10.1016/j.addma.2017.12.004 , open hardware source code: https://osf.io/m2zqk/

The open source syringe pump is also designed in OpenSCAD to be parametric and thus all the components that hold the syringe can be adjusted there and reprinted following the guidelines in [48] and [49]. In addition, even if researchers are using a material that needs either a smaller or larger stepper motor to drive the syringe pump, free and open source Franklin control software [44,45] can accommodate it and the appropriate plastic parts can be printed to hold it following the same use of the parametric OpenSCAD code [49]. Finally, if a much larger syringe is needed the geometric limitations of the Prusa will demand moving to a different FFF machine as discussed in the next section.

Implementing this technology onto a 3-D printer allows for quick adjustments to be made to the slot die in the z-direction and for the slot die to work as an additive manufacturing tool itself. Conventional slot die systems require mechanical alterations to adjust the orientation of the slot die head. The 3-D printing method allows a user to transition between various substrates without difficulty. Finally, it also enables multi-layer (true additive manufacturing) to take place with a slot die system.

\subsection{Limitations and Future Work}

Future work is needed to compare the properties of the materials, materials efficiency and deposition rate of the slot die system used here and with other deposition techniques such as spin coating. This is complicated because the volume used in spin coating depends on the surface tension of the liquid, the thickness desired, and the substrate size. These values are important to calculate degree of solution runoff with a particular rotation speed and duration. In addition, an important difference between slot die and spin coating is the drying kinetics experienced by the solution during deposition. In spin coating, the rotation causes much of the deposited material to be spun-off, which also accelerates the solvent evaporation [5]. While with slot die coating, the solution is deposited and is relatively stable and non-moving, allowing less material to be deposited and evaporation rates to be more controlled, especially if heating elements are added. Furthermore, spin-coating must be done with a circular substrate (or face non-uniformity due to edge beads, geometrical effects, and Bernoulli effects), while slot dies can utilize a rectangular substrate design. This allows for more of the substrate to be utilized for building devices, which further reduces waste.

The constructed slot die is limited to the deposition of films onto smaller substrates. Inner geometries for one size of slot die vary from larger dies. Slot dies with uniform cross sections, such as the one constructed in this experiment, are not capable of maintaining constant pressure over wider webs [83]. In addition, the impact of the geometry of the part needs further study to determine the limitations of FFF for manufacturing [84]. Wider slot dies typically employ a “coat hanger” manifold where the cross-sectional area decreases towards the ends of die. The bed size of the Athena 3-D printer, at 1,586 cubic $\mathrm{cm}$, limits the size of the die. If scaling up were to be pursued, a larger printer would need to be accessed. Alternative open source 3-D printers include various open source Lulzbot [66] and Gigabot [85] models. With some models approaching a build volume of 269,040 cubic $\mathrm{cm}$, the technology to scale up 3-D printed slot dies is readily available. This technology allows for multiple layers to be deposited onto a substrate, further increasing the versatility of the integrated slot die.

In the future, larger slot dies with the appropriate geometries should be constructed. More filaments types could be tested for longer durations, raising the confidence of the die's lifetime in industry 
L.Y. Beeker, Adam M. Pringle, Joshua M. Pearce. Open-source parametric 3-D printed slot die system for thin film semiconductor processing. Additive

Manufacturing 20 (2018) 90-100. https://doi.org/10.1016/j.addma.2017.12.004 , open hardware source code: https://osf.io/m2zqk/

solutions. This slot die was also made capable with $1 \mathrm{~mL}$ volume syringes for proof of concept purposes. Alterations to the code would be required to make the die compatible with larger syringes.

\section{Conclusions}

This study demonstrates that functional lab-grade slot dies may be 3-D printed using low-cost open source hardware methods. Slot dies were implemented into a modified 3-D printer after an appropriate slot die polymer was identified. A case study using $\mathrm{NiO}_{2}$ found an RMS value $0.486 \mathrm{~nm}$, thickness of 17 to $49 \mathrm{~nm}$, and a maximum optical transmission of 99.1\%. This additive manufacturing approach has been shown to be a viable process to produce polymer-based slot dies as well as viable layers of advanced electronic materials, coatings and layered composites. Using this method, a cost savings of over 17,000\% was obtained when compared to commercial slot die systems for laboratories. The open source parametric model provided here of the slot die allows for basic geometries to be altered and customized for a given experiment and printed in a few hours. This process will be useful to researchers studying thin films, while also creating the potential for low cost, scaled-up manufacturing.

\section{References}

1. Beguin AE, (1954) Inventor. Method of coating strip material. US patent No. 2,681,6941954.

2. Hösel M, Angmo D, Søndergaard RR, dos Reis Benatto GA, Carlé JE, Jørgensen M, Krebs FC (2014) High-Volume Processed, ITO-Free Superstrates and Substrates for Roll-to-Roll Development of Organic Electronics. Advanced Science 1(1):n/a-n/a. doi:10.1002/advs.201400002

3. Chang YR, Chang HM, Lin CF, Liu TJ, Wu PY (2007) Three minimum wet thickness regions of slot die coating. Journal of Colloid and Interface Science 308(1):222-230. doi:10.1016/j.jcis.2006.11.054

4. Pederson LR, Singh P, Zhou XD (2006) Application of vacuum deposition methods to solid oxide fuel cells. Vacuum 80(10):1066-1083. doi:10.1016/j.vacuum.2006.01.072

5. Galagan Y, Fledderus H, Gorter H, 't Mannetje HH, Shanmugam S, Mandamparambil R et al (2015) Roll-to-Roll Slot-Die Coated Organic Photovoltaic (OPV) Modules with High Geometrical Fill Factors. Energy Technology 3(8):834-842. doi:10.1002/ente.201500150

6. Krebs FC (2009) Polymer solar cell modules prepared using roll-to-roll methods: Knife-overedge coating, slot-die coating and screen printing. Solar Energy Materials and Solar Cells 93(4):465-475. doi:10.1016/j.solmat.2008.12.012 
L.Y. Beeker, Adam M. Pringle, Joshua M. Pearce. Open-source parametric 3-D printed slot die system for thin film semiconductor processing. Additive Manufacturing 20 (2018) 90-100. https://doi.org/10.1016/j.addma.2017.12.004 , open hardware source code: https://osf.io/m2zqk/

7. Schmitt M, Baunach M, Wengeler L, Peters K, Junges P, Scharfer P, Schabel W (2013). Slotdie processing of lithium-ion battery electrodes-Coating window characterization. Chemical Engineering and Processing: Process Intensification 68:32-37.

doi:10.1016/j.cep.2012.10.011

8. Steenberg T, Hjuler HA, Terkelsen C, Sánchez MTR, Cleemann LN, Krebs FC (2012) Rollto-roll coated PBI membranes for high temperature PEM fuel cells. Energy \& Environmental Science 5(3):6076-6080. doi:10.1039/C2EE02936G

9. Kistler SF, Schweizer PM, (eds) (1997) Liquid Film Coating. Dordrecht: Springer, Netherlands.

10. Korkut I, Kasap M, Ciftci I, Seker U (2004) Determination of optimum cutting parameters during machining of AISI 304 austenitic stainless steel. Materials \& Design 25(4):303-305. doi:10.1016/j.matdes.2003.10.011

11. FOM Technologies (2016) available http://www.allied-dies.com. Accessed 24 October 2016.

12. Ossila (2016) Behind the Scenes - Slot Die Coater Development https://www.ossila.com/blogs/news. Accessed 24 October 2016

13. Gibb A, Abadie S (2014) Building open source hardware: DIY manufacturing for hackers and makers. Pearson Education

14. Jones R, Haufe P, Sells E, Iravani P, Olliver V, Palmer C, Bowyer A (2011) RepRap - the replicating rapid prototype. Robotica 29:177-191. doi:10.1017/S026357471000069X

15. Sells E, Smith Z, Bailard S, Bowyer A, Olliver V (2010) RepRap: The Replicating Rapid Prototyper: Maximizing Customizability by Breeding the Means of Production. In: Piller FT, Tseng MM (eds) Handbook of Research in Mass Customization and Personalization: Strategies and concepts, Vol.1. World Scientific, pp 568-580

16. Bowyer A (2014) 3D printing and humanity's first imperfect replicator. 3D Printing and Additive Manufacturing 1(1): 4-5. doi:10.1089/3dp.2013.0003

17. Anzalone GC, Wijnen B, Pearce JM (2015) Multi-material additive and subtractive prosumer digital fabrication with a free and open-source convertible delta RepRap 3-D printer. Rapid Prototyping Journal 21(5): 506-519. doi:10.1108/RPJ-09-2014-0113

18. Zhang C, Wijnen B, Pearce JM (2016) Open-source 3-D Platform for Low-cost Scientific Instrument Ecosystem. Journal of Laboratory Automation 21(4): 517-525. doi:10.1177/2211068215624406 
L.Y. Beeker, Adam M. Pringle, Joshua M. Pearce. Open-source parametric 3-D printed slot die system for thin film semiconductor processing. Additive Manufacturing 20 (2018) 90-100. https://doi.org/10.1016/j.addma.2017.12.004 , open hardware source code: https://osf.io/m2zqk/

19. OpenSCAD (2016) available http://www.openscad.org. Accessed 24 October 2016.

20. Lin CF, Wong DSH, Liu TJ, Wu PY (2010) Operating windows of slot die coating: Comparison of theoretical predictions with experimental observations. Adv. Polymer Technology 29(1):31-44. doi:10.1002/adv.20173

21. Harris, T.A., Mukherjee, R. and Walczyk, D.(2002). A New Method of Patterning Fluids onto Substrates using a Variable Lip Slot Die. Doi:10.1.1.196.3292

22. Chang, H.M., Chang, Y.R., Lin, C.F. and Liu, T.J., (2007). Comparison of vertical and horizontal slot die coatings. Polymer Engineering \& Science, 47(11), pp.1927-1936. DOI: 10.1002/pen.20910

23. Chang, Y.R., Lin, C.F. and Liu, T.J., (2009). Start-up of slot die coating. Polymer Engineering \& Science, 49(6), pp.1158-1167. DOI: 10.1002/pen.21360

24. Bhamidipati, K.L. and Harris, T.A., (2009), January. Numerical Analysis of the Effects of Processing Conditions on the Casting of High Temperature PEMFC Membrane Solutions. In ASME 2009 7th International Conference on Fuel Cell Science, Engineering and Technology (pp. 695-702). American Society of Mechanical Engineers. doi:10.1115/FuelCell2009-85064

25. Anazawa, T., Fujisawa, N., Yajima, T. and Yamagata, T., (2010). Vortex formation in a viscoelastic entry flow of asymmetric planar contraction. Journal of visualization, 13(3), pp.191-193. DOI: 10.1007/s12650-010-0028-z

26. Bhamidipati, K., Didari, S. and Harris, T.A., (2012). Experimental study on air entrainment in slot die coating of high-viscosity, shear-thinning fluids. Chemical engineering science, 80 , pp.195-204. Doi: 10.1016/j.ces.2012.06.033

27. Lee KY, Liu TJ (1989). Design and analysis of a dual-cavity coat-hanger die. Polymer Engineering \& Science 29(15): 1066-1075. doi: 10.1002/pen.760291513

28. Helmy H (1987). Aspects of the design of coathanger dies for cast film and sheet applications. Advances in Polymer Technology 7(1): 59-69. doi:10.1002/adv.1987.060070107

29. Ahn WG, Lee SH, Nam J, Jung HW (2015) Effect of flow rate variation on the frequency response in slot coating process with different upstream sloped die geometries. Korean Journal of Chemical Engineering 32(7): 1218-1221. doi:10.1007/s11814-015-0079-X

30. Wohlers T. (2016) Wohlers Report 2016. Wohlers Associates, Inc; 2016 Apr 10. 
L.Y. Beeker, Adam M. Pringle, Joshua M. Pearce. Open-source parametric 3-D printed slot die system for thin film semiconductor processing. Additive Manufacturing 20 (2018) 90-100. https://doi.org/10.1016/j.addma.2017.12.004 , open hardware source code: https://osf.io/m2zqk/

31. Anzalone GC, Zhang C, Wijnen B, Sanders PG, Pearce JM (2013) A low-cost open-source metal 3-D printer. IEEE Access 1:803-810. doi:10.1109/ACCESS.2013.2293018

32. Haselhuhn AS, Gooding EJ, Glover AG, Anzalone GC, Wijnen B, Sanders PG, Pearce JM (2014) Substrate release mechanisms for gas metal arc weld 3D aluminum metal printing. 3D Printing and Additive Manufacturing 1(4):204-209. doi:10.1089/3dp.2014.0015

33. Yuenyong N, Haselhuhn A, Wijnen B, Sanders PG, Pearce JM (2015) Integrated VoltageCurrent Monitoring and Control of Gas Metal Arc Weld Magnetic Ball-Jointed Open Source 3-D Printer. Machines 3(4):339-351. doi:10.3390/machines3040339

34. Haselhuhn AS, Wijnen B, Anzalone GC, Sanders PG, Pearce JM (2015) In situ formation of substrate release mechanisms for gas metal arc weld metal 3-D printing. Journal of Materials Processing Technology 226:50-59. doi:10.1016/j.jmatprotec.2015.06.038

35. Haselhuhn AS, Buhr MW, Wijnen B, Sanders PG, Pearce JM (2016) Structure-Property Relationships of Common Aluminum Weld Alloys Utilized as Feedstock for GMAW-based 3D Metal Printing. Materials Science and Engineering 673:511-523.

doi:10.1016/j.msea.2016.07.099

36. Wang JT-W, Ball JM, Barea EM, Abate A, Alexander-Webber JA, Huang J, Saliba M, MoraSero I, Bisquert J, Snaith HJ, Nicholas RJ (2014) Low-Temperature Processed Electron Collection Layers of Graphene/TiO2 Nanocomposites in Thin Film Perovskite Solar Cells. Nano Letters 14(2):724-730.

37. Manders JR, Tsang SW, Hartel MJ, Lai TH, Chen S, Amb CM, Reynolds JR, So F (2013) Solution-Processed Nickel Oxide Hole Transport Layers in High Efficiency Polymer Photovoltaic Cells. Advanced Functional Materials 23(23):2993-3001.

38. Barlow, P., 1814. New mathematical tables.

39. Aerocom. Barlow's Formula Calculator (2016) Barlow's Formula Calculator available http://www.aerocomfittings.com/barlows.html.

40. Curbell Plastics (2016) PETG available https://www.curbellplastics.com/ResearchSolutions/Materials/PETG

41. Crone KP (2016) Slot Die Coating Principal and Application. Accessed 16 December 2016.

42. Athena Build Overview. http://www.appropedia.org/Athena Build Overview. Accessed October 242016 
L.Y. Beeker, Adam M. Pringle, Joshua M. Pearce. Open-source parametric 3-D printed slot die system for thin film semiconductor processing. Additive Manufacturing 20 (2018) 90-100. https://doi.org/10.1016/j.addma.2017.12.004 , open hardware source code: https://osf.io/m2zqk/

43. Cura (2016) available https://ultimaker.com/en/products/cura-software Accessed 24 October 2016.

44. Wijnen B, Anzalone GC, Haselhuhn AS, Sanders PG, Pearce JM (2016) Free and opensource control software for 3-D motion and processing. Journal of Open Research Software 4(1):e2. doi:10.1016/j.msea.2016.07.099

45. Franklin (2016) available https://github.com/mtu-most/franklin. Accessed October 242016

46. ImageJ (2016) available https://imagej.nih.gov/ij/. Accessed December 152016

47. Prusa http://reprap.org/wiki/Prusa Mendel (iteration 1). Accessed October 242016

48. Wijnen B, Hunt EJ, Anzalone GC, Pearce JM (2014) Open-source syringe pump library. PLoS One 9(9):e107216. doi:10.1371/journal.pone.0107216

49. Pearce JM, Anzalone NC, Heldt CL (2016) Open-Source Wax RepRap 3-D Printer for Rapid Prototyping Paper-Based Microfluidics. Journal of Laboratory Automation 21(4):510-516. doi:10.1177/2211068215624408

50. Soonmin H (2016) Preparation and Characterization of Nickel Oxide Thin Films: A Review. International Journal of Applied Chemistry 12(2):87-93.

51. Open source 3D printable transmittance tool https://osf.io/etx72/ Accessed October 262016

52. Bernasconi R, Natale G, Levi M, Magagnin L (2015) Electroless Plating of PLA and PETG for 3D Printed Flexible Substrates. ECS Transactions 66:23-35. doi:10.1149/06619.0023ecst

53. Sharon C, Sharon M (2012) Studies on Biodegradation of Polyethylene terephthalate: A synthetic polymer. J. Microbiology Biotechnology Research 2(2):248-257.

54. Tokiwa, Y, Calabia, BP, Ugwu CU, Aiba S (2009) Biodegradability of Plastics. International Journal of Molecular Sciences 10:3722-3742. doi:10.3390/ijms10093722

55. Vert M, Schwarch G, Coudane J (1995) Present and Future of PLA Polymers. Journal of Macromolecular Science 32:787-796. doi:10.1080/10601329508010289

56. Carneiro OS, Silva AF, Gomes R (2015) Fused deposition modeling with polypropylene. Materials \& Design 83:768-776. doi:10.1016/j.matdes.2015.06.053 
L.Y. Beeker, Adam M. Pringle, Joshua M. Pearce. Open-source parametric 3-D printed slot die system for thin film semiconductor processing. Additive Manufacturing 20 (2018) 90-100. https://doi.org/10.1016/j.addma.2017.12.004 , open hardware source code: https://osf.io/m2zqk/

57. Open source 3-D printable slot die https://osf.io/m2zqk/ Accessed October 262016

58. Steirer KX, Chesin JP, Widjonarko NE, Berry JJ, Miedaner A, Ginley D.S. Olson DC (2010) Solution deposited $\mathrm{NiO}$ thin-films as hole transport layers in organic photovoltaics. Organic Electronics 11(8):1414-1418. doi:10.1016/j.orgel.2010.05.008

59. Pereira S, Gonçalves A, Correia N, Pinto J, Pereira L, Martins R, Fortunato E (2014) Electrochromic behavior of $\mathrm{NiO}$ thin films deposited by e-beam evaporation at room temperature. Solar Energy Materials and Solar Cells 120:109-115. doi:10.1016/j.solmat.2013.08.024

60. Manders JR, Tsang SW, Hartel MJ, Lai TH, Chen S, Amb CM, Reynolds JR, So F (2013) Solution-Processed Nickel Oxide Hole Transport Layers in High Efficiency Polymer Photovoltaic Cells. Advanced Functional Materials 23:2993-3001. doi:10.1002/adfm.201202269

61. Patil PS, Kadam LD (2002) Preparation and characterization of spray pyrolyzed nickel oxide (NiO) thin films. Applied Surface Science 199:211-221. doi:10.1016/S0169-4332(02)008395

62. Jlassi M, Sta I, Hajji M, Ezzaouia H (2014) Optical and electrical properties of nickel oxide thin films synthesized by sol-gel spin coating. Materials Science in Semiconductor Processing 21:7-13. doi:10.1016/j.mssp.2014.01.018

63. Al-Ghamdi AA, Mahmoud WE, Yaghmour SJ, Al-Marzouki FM (2009) Structure and optical properties of nanocrystalline $\mathrm{NiO}$ thin film synthesized by sol-gel spin-coating method. Journal of Alloys and Compounds 486:9-13. doi:10.1016/j.jallcom.2009.06.139

64. Irwin MD, Buchholz DB, Hains AW, Chang RPH, Marks TJ (2008) p-Type semiconducting nickel oxide as an efficiency-enhancing anode interfacial layer in polymer bulkheterojunction solar cells. Proceedings of the National Academy of Sciences 105:2783-2787. doi:10.1073/pnas.0711990105

65. Jiang DY, Qin JM, Wang X, Gao S, Liang QC, Zhao JX (2012) Optical properties of NiO thin films fabricated by electron beam evaporation. Vacuum 86:1083-1086.

doi:10.1016/j.vacuum.2011.10.003

66. Aleph Objects. Lulzbot Taz 6. https://www.lulzbot.com/store/printers/lulzbot-taz-6 Accessed 26 October 2017.

67. The Maker Map. Available online: http://themakermap.com/ Accessed 26 October 2017. 68. Hackerspaces. Available online: https://wiki.hackerspaces.org/ Accessed 26 October 2017. 
L.Y. Beeker, Adam M. Pringle, Joshua M. Pearce. Open-source parametric 3-D printed slot die system for thin film semiconductor processing. Additive Manufacturing 20 (2018) 90-100. https://doi.org/10.1016/j.addma.2017.12.004 , open hardware source code: https://osf.io/m2zqk/

69. Stacey, M. (2014). The FAB LAB network: A global platform for digital invention, education and entrepreneurship. Innovations, 9, 221-238.

70. Fab Foundation. Fab Labs. Available online: http://www.fabfoundation.org/fab-labs/ Accessed 26 October 2017.

71. Massis, B. E. (2013). 3D printing and the library. New Library World, 114(7/8), 351-354.

72. Moorefield-Lang, H. (2014) 3-D printing in your libraries and classrooms. Knowl. Quest, 43, 70-73.

73. Pearce, J. (2012) Building Research Equipment with Free, Open-Source Hardware. Science, 337, 1303-1304.

74. Pearce, J. Open-Source Lab: How to Build Your Own Hardware and Reduce Research Costs, 1st ed.; Elsevier: Waltham, MA, USA, 2014.

75. Baden, T., Chagas, A., Marzullo, T., Prieto-Godino, L., Euler, T. (2015) Open Labware: 3-D Printing Your Own Lab Equipment. PLoS Biol., 13, e1002086.

76. Gill, J. M., \& Hart, A. S. (2016). Opening New Frontiers in the Development of Life Sciences Technology with Collaborative 3D Printing Technology. Journal of Laboratory Automation, 21(4), 487-488.

77. Pearce, J. M. (2017). Emerging Business Models for Open Source Hardware. Journal of Open Hardware, 1(1).

78. Dryden, M. D., Fobel, R., Fobel, C., \& Wheeler, A. R. (2017). Upon the Shoulders of Giants: Open-Source Hardware and Software in Analytical Chemistry. Analytical Chemistry, 89(8), 4330-4338.

79. Rifkin, J. (2014). The zero marginal cost society: The internet of things, the collaborative commons, and the eclipse of capitalism. St. Martin's Press.

80. Wittbrodt, B. T., Glover, A. G., Laureto, J., Anzalone, G. C., Oppliger, D., Irwin, J. L., \& Pearce, J. M. (2013). Life-cycle economic analysis of distributed manufacturing with opensource 3-D printers. Mechatronics, 23(6), 713-726.

81. Petersen, E.E., Pearce, J. (2017). Emergence of Home Manufacturing in the Developed World: Return on Investment for Open-Source 3-D Printers. Technologies, 5, 7, doi:10.3390/technologies5010007

82. Make XYZ Available online: https://www.makexyz.com/ $\underline{\text { Accessed } 26 \text { October } 2017 .}$

83. Na SY, Kim D H (1995) Three-dimensional modelling of Non-Newtonian fluid flow in a coat-hanger die. Korean Journal of Chemical Engineering 12(2):236-243.

doi:10.1007/BF02705652

84. Fernandez-Vicente M, Canyada M, Conejero, A (2015) Identifying limitations for design for manufacturing with desktop FFF 3D printers. International Journal of Rapid Manufacturing 5(1):116-128. doi:10.1504/IJRAPIDM.2015.073551

85. re:3D Inc. (2016) available https://re3d.org/. Accessed 24 October 2016 